\title{
A REMARK ON MAXIMAL OPERATORS ALONG DIRECTIONS IN $\mathbb{R}^{2}$
}

\author{
Angeles Alfonseca, Fernando Soria, and Ana Vargas
}

ABSTRACT. In this paper we give a simple proof of a long-standing conjecture, recently proved by N. Katz, on the weak-type norm of a maximal operator associated with an arbitrary collection of directions in the plane. The proof relies upon a geometric argument and on induction with respect to the number of directions. Applications are given to estimate the behavior of several types of maximal operators.

\section{Introduction}

Let $\Omega$ be a subset of $\left[0, \frac{\pi}{4}\right)$. Associated to it, we define the maximal operator $M_{\Omega}$ acting on locally integrable functions $f$ on $\mathbb{R}^{2}$ by

$$
M_{\Omega} f(x)=\sup _{x \in R \in \mathcal{B}_{\Omega}} \frac{1}{|R|} \int_{R}|f(y)| d y,
$$

where $\mathcal{B}_{\Omega}$ denotes the basis of all rectangles with longest side forming an angle $\alpha$ with the x-axis, for some $\alpha \in \Omega$.

For finite sets $\Omega$, it was conjectured that the $L^{2}$ norm of $M_{\Omega}$ had logarithmic growth with respect to the cardinality, $N$, of $\Omega$. More precisely, that $M_{\Omega}$ satisfied the following estimate

$$
\left\|M_{\Omega} f\right\|_{L^{2}\left(\mathbb{R}^{2}\right)} \leq C(\log N)^{\beta}\|f\|_{L^{2}\left(\mathbb{R}^{2}\right)},
$$

for some exponent $\beta$ and with $C$ independent of $\Omega$ and $f$.

This inequality was first proved in the seventies for some special sets $\Omega$, such as uniformly distributed directions (see [9]). Also, for lacunary sequences the result holds with $\beta=0$ (see [4] and [7]) and therefore one can take an infinite sequence in this case. In 1995, Barrionuevo [3] obtained the following result:

$$
\left\|M_{\Omega} f\right\|_{L^{2}\left(\mathbb{R}^{2}\right)} \leq C N^{2 / \sqrt{\log N}}\|f\|_{L^{2}\left(\mathbb{R}^{2}\right)}
$$

again for card $\Omega=N<\infty$ and $C$ independent of $\Omega$ and $f$.

Conjecture (1) for general finite sets $\Omega$ was finally proved by Katz in 1999, [6], (see also [5]), with the best possible exponent, that is, with $\beta=1$.

Received July 10, 2001.

2000 Mathematics Subject Classification. 42B25.

Key words and phrases. maximal operators, weak-type estimates.

Research partially supported by EC grant via the network "Harmonic Analysis and related problems" and by grant BFM2001-0189. 
The main result of this paper (Theorem 1 below) establishes an estimate for the weak type $(2,2)$ of an operator in a related problem which gives, as a consequence, a simple proof of that conjecture. With our arguments we do not obtain the sharp exponent of the logarithm given in Katz' proof. Theorem 1 however does provide a wide range of applications which we explore in the last section. The proof of Theorem 1 uses some geometric arguments, in the same spirit of the work of Strömberg, [9], and Córdoba and Fefferman, [4].

In order to state our result, we introduce first some notation. Given the set $\Omega \subset\left[0, \frac{\pi}{4}\right)$ we consider a subset $\Omega_{0}=\left\{\theta_{l}: l=1,2, \ldots\right\} \subset \Omega$ with $\frac{\pi}{4}>\theta_{1}>$ $\theta_{2}>\ldots>\theta_{l}>\ldots$ There is no restriction on whether $\Omega_{0}$ is finite or not. Let us define for $l=1,2, \ldots, \Omega_{l}=\left\{\alpha \in \Omega: \theta_{l} \leq \alpha<\theta_{l-1}\right\}$, where we have set $\theta_{0}=\frac{\pi}{4}$. We shall assume that $\Omega_{0}$ is chosen so that $\Omega=\cup_{l \geq 1} \Omega_{l}$. To each set $\Omega_{l}, l=0,1,2, \ldots$ we associate the corresponding basis $\mathcal{B}_{l} \subset \overline{\mathcal{B}}_{\Omega}$. We define the maximal operators associated to each $\Omega_{l}$ as

$$
M_{\Omega_{l}} f(x)=\sup _{x \in R \in \mathcal{B}_{l}} \frac{1}{|R|} \int_{R}|f(y)| d y, \quad l=0,1,2, \ldots
$$

In the next section we shall prove the following

Theorem 1. There exist constants $C_{1}, C_{2}$ independent of $\Omega$ such that

$$
\left\|M_{\Omega}\right\|_{L^{2} \rightarrow L^{2, \infty}}^{2} \leq C_{1} \sup _{1 \leq l}\left\|M_{\Omega_{l}}\right\|_{L^{2} \rightarrow L^{2, \infty}}^{2}+C_{2}\left\|M_{\Omega_{0}}\right\|_{L^{2} \rightarrow L^{2, \infty}}^{2},
$$

where $\|T\|_{L^{2} \rightarrow L^{2, \infty}}$ denotes the "weak type $(2,2)$ " norm of the operator $T$.

What the theorem says is that if we decompose $\Omega$ into disjoint blocks, "separated" by the elements of certain collection of directions $\Omega_{0}$, then the price that one pays to make the inequality

$$
\left\|\sup _{l} M_{\Omega_{l}}\right\|_{L^{2} \rightarrow L^{2, \infty}} \leq C \sup _{l}\left\|M_{\Omega_{l}}\right\|_{L^{2} \rightarrow L^{2, \infty}}
$$

true is given by the norm of the operator $M_{\Omega_{0}}$, associated to $\Omega_{0}$, in the precise form stated above. This can be seen as a weak- $L^{2}$ quasi-orthogonality principle for the family of operators $\left\{M_{\Omega_{j}}\right\}$. In particular, if the "separating" set $\Omega_{0}$ is given by a lacunary sequence, then (3) is true no matter how the "intermediate" sets $\Omega_{l}, l=1,2, \ldots$ are chosen. This extends a result by Sjögren and Sjölin [8]. In Section 3 we present this and some other applications of our theorem, such as the new proof of Katz's theorem that we mentioned above.

\section{Proof of the main result}

Let us start by changing slightly our previous hypotheses. Given a set $\Omega \subset$ $\left[0, \frac{\pi}{4}\right)$, we consider the basis $\mathcal{B}_{\Omega}$ of all parallelograms with the shorter sides parallel to the y-axis and the longer sides forming an angle $\alpha$ with the x-axis, for some $\alpha \in \Omega$. With certain abuse of language, we shall call these parallelograms "rectangles".

We introduce now the following notation: given a rectangle $R \in \mathcal{B}_{\Omega}, P_{1}(R)$ will denote the projection of $R$ on the x-axis. If $P_{1}(R)=\left[a_{R}^{1}, a_{R}^{2}\right]$, we also define 
$P_{2,1}(R)=\left\{y:\left(a_{R}^{1}, y\right) \in R\right\}$ and $P_{2,2}(R)=\left\{y:\left(a_{R}^{2}, y\right) \in R\right\} . P_{2,1}(R)$ and $P_{2,2}(R)$ are the projections of the two "vertical sides" of $R$ on the y-axis. Note that $\left|P_{2,1}(R)\right|=\left|P_{2,2}(R)\right|$ and $|R|=\left|P_{1}(R)\right| \cdot\left|P_{2,1}(R)\right|$.

Let $\Omega_{0}$ be any ordered subset of $\Omega$. By a simple limiting argument, it is clear that in order to prove (2) we may assume with no loss of generality that $\Omega$ (and, hence, $\Omega_{0}$ too) is finite. We shall denote the elements of $\Omega_{0}$ by $\theta_{1}, \theta_{2}, \ldots, \theta_{N}$, with

$$
\frac{\pi}{4}=\theta_{0}>\theta_{1}>\theta_{2}>\ldots>\theta_{N-1}>\theta_{N} \geq 0 .
$$

Define $\Omega_{l}=\left\{\alpha \in \Omega: \theta_{l} \leq \alpha<\theta_{l-1}\right\}$, for $l=1, \ldots, N$. Then $\Omega=\cup_{l=1}^{N} \Omega_{l}$ by assuming simply that $\theta_{N}=\min \Omega$. To each set $\Omega_{l}, l=0,1, \ldots, N$ we associate the corresponding basis $\mathcal{B}_{l} \subset \mathcal{B}_{\Omega}$.

We now define the maximal operators

$$
M_{\Omega} f(x)=\sup _{x \in R \in \mathcal{B}_{\Omega}} \frac{1}{|R|} \int_{R}|f(y)| d y
$$

and

$$
M_{\Omega_{l}} f(x)=\sup _{x \in R \in \mathcal{B}_{l}} \frac{1}{|R|} \int_{R}|f(y)| d y, \quad l=0,1, \ldots, N .
$$

To prove (2) we look at the level sets of $M_{\Omega}$. If $x \in\left\{M_{\Omega} f(x)>\lambda\right\}$, there is a rectangle $R_{x} \in \mathcal{B}_{\Omega}$ containing $x$ such that

$$
\frac{1}{\left|R_{x}\right|} \int_{R_{x}}|f(y)| d y>\lambda
$$

and therefore

$$
\left\{M_{\Omega} f(x)>\lambda\right\} \subset \bigcup_{x \in\left\{M_{\Omega} f(x)>\lambda\right\}} R_{x} .
$$

So if we consider a compact set $K \subset\left\{M_{\Omega} f(x)>\lambda\right\}$, then $K \subset \bigcup_{j=1}^{s} R_{x_{j}}$ for some finite family of rectangles $\mathcal{F}=\left\{R_{x_{j}}\right\}_{j=1}^{s}$ satisfying (4).

From the family $\mathcal{F}$ we select a subfamily $\overline{\mathcal{F}}=\left\{B_{k}\right\}$ in the following way: we take $B_{1}$ as the rectangle $R \in \mathcal{F}$ with longest projection on the x-axis. Assuming we have already chosen $B_{1}, \ldots, B_{n-1}$, we take $B_{n}$ as the rectangle $R$ of the remaining collection $\mathcal{F} \backslash\left\{B_{k}\right\}_{j=1}^{n-1}$ such that $\left|P_{1}(R)\right|$ is maximal among the rectangles satisfying

$$
\sum_{k=1}^{n-1}\left|R \cap B_{k}\right| \leq \frac{1}{2}|R| .
$$

It is easy to see that the family $\left\{B_{k}\right\}$ has the following two properties:

$$
\begin{gathered}
\sum\left|B_{k}\right| \leq 2\left|\cup B_{k}\right| \\
\int\left(\sum \chi_{B_{k}}\right)^{2} \leq 2 \sum\left|B_{k}\right| .
\end{gathered}
$$


In order to estimate the weak type $(2,2)$ norm for $M_{\Omega}$, we first observe that

$$
\begin{gathered}
\sum\left|B_{k}\right| \leq \frac{1}{\lambda} \sum \int_{B_{k}}|f| \leq \frac{1}{\lambda}\|f\|_{2}\left\|\sum \chi_{B_{k}}\right\|_{2} \leq \\
\leq \frac{\sqrt{2}}{\lambda}\|f\|_{2}\left(\sum\left|B_{k}\right|\right)^{1 / 2},
\end{gathered}
$$

where we have used (6). This implies

$$
\left(\sum\left|B_{k}\right|\right)^{1 / 2} \leq \frac{\sqrt{2}}{\lambda}\|f\|_{2}
$$

If we show that

$$
\left|\cup R_{x_{j}} \backslash \cup B_{k}\right| \leq c_{0} \sum\left|B_{k}\right|
$$

then, using (7) we get

$$
\begin{gathered}
|K| \leq\left|\cup R_{x_{j}}\right| \leq\left|\cup B_{k}\right|+\left|\cup R_{x_{j}} \backslash \cup B_{k}\right| \leq \\
\quad \leq\left(1+c_{0}\right)\left(\sum\left|B_{k}\right|\right) \leq \frac{2\left(1+c_{0}\right)}{\lambda^{2}}\|f\|_{2}^{2} .
\end{gathered}
$$

Consequently, we would obtain

$$
\left|\left\{M_{\Omega} f(x)>\lambda\right\}\right| \leq \frac{2\left(1+c_{0}\right)}{\lambda^{2}}\|f\|_{2}^{2},
$$

provided $c_{0}$ is independent of the compact set $K$.

It remains to prove then (8). Let $R$ be one of the rectangles in $\mathcal{F} \backslash \overline{\mathcal{F}}$. Then

$$
\sum_{B_{k}:\left|P_{1}\left(B_{k}\right)\right| \geq\left|P_{1}(R)\right|}\left|R \cap B_{k}\right|>\frac{1}{2}|R| .
$$

If $R \in \mathcal{B}_{l}$, we observe that one of the following inequalities must hold:

$$
\sum_{B_{k} \in \mathcal{B}_{l}:\left|P_{1}\left(B_{k}\right)\right| \geq\left|P_{1}(R)\right|} \frac{\left|R \cap B_{k}\right|}{|R|}>\frac{1}{4},
$$

or

$$
\sum_{B_{k} \notin \mathcal{B}_{l}:\left|P_{1}\left(B_{k}\right)\right| \geq\left|P_{1}(R)\right|} \frac{\left|R \cap B_{k}\right|}{|R|}>\frac{1}{4} .
$$

Let us denote

$$
\mathcal{F}_{1}=\bigcup_{l}\left\{R \in(\mathcal{F} \backslash \overline{\mathcal{F}}) \cap \mathcal{B}_{l}: \sum_{B_{k} \in \mathcal{B}_{l}:\left|P_{1}\left(B_{k}\right)\right| \geq\left|P_{1}(R)\right|} \frac{\left|R \cap B_{k}\right|}{|R|}>\frac{1}{4}\right\},
$$

and

$$
\mathcal{F}_{2}=\bigcup_{l}\left\{R \in(\mathcal{F} \backslash \overline{\mathcal{F}}) \cap \mathcal{B}_{l}: \sum_{B_{k} \notin \mathcal{B}_{l}:\left|P_{1}\left(B_{k}\right)\right| \geq\left|P_{1}(R)\right|} \frac{\left|R \cap B_{k}\right|}{|R|}>\frac{1}{4}\right\} .
$$


Observe that if $R \in \mathcal{B}_{l}$ and (9) holds, then $R \subset\left\{x: M_{\Omega_{l}}\left(\sum_{B_{k} \in \mathcal{B}_{l}} \chi_{B_{k}}\right)>\frac{1}{4}\right\}$. Hence,

$$
\begin{aligned}
& \left|\cup_{\mathcal{F}_{1}} R\right| \leq \sum_{l=1}^{N}\left|\left\{M_{\Omega_{l}}\left(\sum_{B_{k} \in \mathcal{B}_{l}} \chi_{B_{k}}\right)>\frac{1}{4}\right\}\right| \leq 16 \sum_{l=1}^{N}\left\|M_{\Omega_{l}}\right\|_{L^{2} \rightarrow L^{2, \infty}}^{2}\left\|\sum_{B_{k} \in \mathcal{B}_{l}} \chi_{B_{k}}\right\|_{2}^{2} \\
& \quad \leq 16 \sup _{l=1, \ldots, N}\left\|M_{\Omega_{l}}\right\|_{L^{2} \rightarrow L^{2, \infty}}^{2}\left\|\sum_{B_{k} \in \overline{\mathcal{F}}} \chi_{B_{k}}\right\|_{2}^{2} .
\end{aligned}
$$

Now, suppose we know that there exists an universal constant $c$ with the property that, for any $R \in \mathcal{F}_{2} \cap \mathcal{B}_{l}$, there are $\widehat{R_{+}}, \widehat{R_{-}} \in \mathcal{B}_{0}$ containing $R$ so that

$$
\frac{\left|B_{k} \cap R\right|}{|R|} \leq c \frac{\left|B_{k} \cap \widehat{R_{+}}\right|}{\left|\widehat{R_{+}}\right|}+c \frac{\left|B_{k} \cap \widehat{R_{-}}\right|}{\left|\widehat{R_{-}}\right|},
$$

for all $B_{k} \notin \mathcal{B}_{l}$. Then, we would have

$$
\begin{gathered}
\left|\cup_{\mathcal{F}_{2}} R\right| \leq\left|\left\{M_{\Omega_{0}}\left(\sum \chi_{B_{k}}\right)>\frac{1}{8 c}\right\}\right| \leq 64 c^{2}\left\|M_{\Omega_{0}}\right\|_{L^{2} \rightarrow L^{2, \infty}}^{2} \cdot\left\|\sum \chi_{B_{k}}\right\|_{2}^{2} \leq \\
\leq 128 c^{2}\left\|M_{\Omega_{0}}\right\|_{L^{2} \rightarrow L^{2, \infty}}^{2}\left|\cup B_{k}\right| .
\end{gathered}
$$

Combining (11) and (13), we get (8) with $c_{0}=32 \sup \left\|M_{\Omega_{l}}\right\|^{2}+128 c^{2}\left\|M_{\Omega_{0}}\right\|^{2}$. So we have to prove (12).

In order to do this, we introduce first some notation.

Definition. Given $U, V \in \mathcal{B}_{\Omega}$, we say that $U$ crosses $V$ entirely if there exists an interval $J$ such that

$$
J \subset P_{1}(U), P_{1}(V),
$$

and, if $S$ is the strip $S=\{(x, y): x \in J\}, \widetilde{U}=U \cap S, \widetilde{V}=V \cap S$, then

$$
\begin{gathered}
\widetilde{U} \cap \widetilde{V} \neq \emptyset, \\
P_{2, i}(\widetilde{U}) \cap P_{2, i}(\widetilde{V})=\emptyset \text { for } i=1,2 .
\end{gathered}
$$

Observe that $U$ crosses entirely $V$ if and only if $V$ crosses entirely $U$.

Lemma 2. If $V_{1}, V_{2}$ cross entirely $U$, with $\left|P_{2,1}\left(V_{1}\right)\right|=\left|P_{2,1}\left(V_{2}\right)\right|$ and $\operatorname{angle}\left(V_{2}, U\right)=\alpha_{2} \leq \alpha_{1}=\operatorname{angle}\left(V_{1}, U\right)$, then

$$
\left|V_{1} \cap U\right| \leq\left|V_{2} \cap U\right| .
$$

Proof. We may assume without loss of generality that $U$ has sides parallel to the axis. Then, if $a=\left|P_{2,1}(U)\right|$ and $b=\left|P_{2,1}\left(V_{j}\right)\right|$,

$$
\left|V_{j} \cap U\right|=\frac{a \cdot b}{\tan \alpha_{j}},
$$

so if $\alpha_{2} \leq \alpha_{1}$, then $\left|V_{1} \cap U\right| \leq\left|V_{2} \cap U\right|$. 
Lemma 3. If $V_{1}, V_{2}$ are parallel, cross entirely $U$ and $\left|P_{1}\left(V_{1}\right)\right|=\left|P_{1}\left(V_{2}\right)\right|=L$, then

$$
\frac{\left|U \cap V_{1}\right|}{\left|V_{1}\right|}=\frac{\left|U \cap V_{2}\right|}{\left|V_{2}\right|}
$$

Proof. Again, we may assume that $U$ has sides parallel to the axis. Set $a=\left|P_{2,1}(U)\right|, \alpha=\operatorname{angle}\left(V_{j}, U\right)$ and $b_{j}=\left|P_{2,1}\left(V_{j}\right)\right|$ for $j=1,2$ (note that $\alpha$ does not depend on $j$ ). Then, as in lemma 2 , we have

$$
\frac{\left|U \cap V_{j}\right|}{\left|V_{j}\right|}=\frac{a \cdot b_{j}}{\tan \alpha} \cdot \frac{1}{b_{j} \cdot L}=\frac{a}{L \tan \alpha},
$$

which does not depend on $j$.

Once we have these two lemmas, let us prove (12). Let $R \in \mathcal{F}_{2} \cap \mathcal{B}_{l}, B \in \overline{\mathcal{F}} \backslash \mathcal{B}_{l}$ such that $B \cap R \neq \emptyset$ and $\left|P_{1}(B)\right| \geq\left|P_{1}(R)\right|$. Let $\alpha_{R}$ be the angle that $R$ forms with the x-axis, and $\alpha_{B}$ the angle that $B$ forms with the x-axis. We shall assume that $\alpha_{B}>\alpha_{R}$. (The case $\alpha_{B}<\alpha_{R}$ can be handled in a similar way.) Then there exists $\theta_{k} \in \Omega_{0}$ such that

$$
\alpha_{B} \geq \theta_{k}>\alpha_{R}
$$

Let $\widetilde{R}$ be the smallest rectangle in the direction of $\theta_{k}$ containing $R$. We define $\widehat{R_{+}}$as the rectangle concentric with $\widetilde{R}$ and 9 times bigger. We will call $\widehat{R}_{\text {mid }}$ to the middle third of $\widehat{R_{+}}$, i.e. the rectangle in the direction of $\theta_{k}$ satisfying

$$
\begin{array}{r}
P_{1}\left(\widehat{R}_{\mathrm{mid}}\right)=P_{1}\left(\widehat{R}_{+}\right), \\
\widetilde{R} \subset \widehat{R}_{\mathrm{mid}}, \\
|\widetilde{R}|=\frac{1}{3}\left|\widehat{R}_{\mathrm{mid}}\right| .
\end{array}
$$

We have to show that

$$
\frac{|B \cap R|}{|R|} \leq c \frac{\left|B \cap \widehat{R_{+}}\right|}{\left|\widehat{R_{+}}\right|} .
$$

Since $\widehat{R_{+}} \in \mathcal{B}_{0}$, this gives (12).

To simplify the notation, from now and on we shall write $\widehat{R}$ instead of $\widehat{R_{+}}$. We define $R^{\infty}$ as the smallest infinite strip containing $R$ and with the same slope. Let $B^{\prime}$ be the smallest rectangle containing $B$ with $P_{1}\left(B^{\prime}\right) \supset P_{1}(\widehat{R})$, and define

$$
B^{*}=B^{\prime} \cap\left[P_{1}(\widehat{R}) \times \mathbb{R}\right] .
$$

Observe that $\left|B^{*} \cap \widehat{R}\right| \leq 3|B \cap \widehat{R}|$.

We shall consider two cases:

Case 1. $B^{*}$ crosses entirely $\widehat{R}$.

Let $R^{r o t}$ be a rectangle in the direction of $\theta_{k}$ such that

$$
P_{1}\left(R^{r o t}\right)=P_{1}(\widehat{R})
$$




$$
\begin{gathered}
R^{r o t} \subset \widehat{R}_{\text {mid }}, \\
\left|P_{2,1}\left(R^{r o t}\right)\right|=\left|P_{2,1}(R)\right| .
\end{gathered}
$$

Then, by Lemma 2,

$$
\left|B^{*} \cap R\right| \leq\left|B^{*} \cap R^{\infty}\right| \leq\left|B^{*} \cap R^{r o t}\right|,
$$

and

$$
\frac{\left|B^{*} \cap R\right|}{|R|} \leq \frac{\left|B^{*} \cap R^{r o t}\right|}{|R|}=\frac{3\left|B^{*} \cap R^{r o t}\right|}{\left|R^{r o t}\right|}=\frac{3\left|B^{*} \cap \widehat{R}\right|}{|\widehat{R}|} .
$$

In the last equality we have used Lemma 3. So we get

$$
\frac{|B \cap R|}{|R|} \leq \frac{\left|B^{*} \cap R\right|}{|R|} \leq \frac{3\left|B^{*} \cap \widehat{R}\right|}{|\widehat{R}|} \leq \frac{9|B \cap \widehat{R}|}{|\widehat{R}|} .
$$

Case 2. $B^{*}$ does not cross entirely $\widehat{R}$.

We may assume that $\left|P_{2,1}\left(B^{*}\right)\right| \leq \frac{1}{3}\left|P_{2,1}(\widehat{R})\right|$, because otherwise we would have

$$
R \subset \widehat{R}_{\text {mid }} \subset 25 B^{*} \subset 125 B .
$$

But if $\left|P_{2,1}\left(B^{*}\right)\right| \leq \frac{1}{3}\left|P_{2,1}(\widehat{R})\right|$, then we have

$$
\left|B^{*}\right| \leq 3\left|B^{*} \cap \widehat{R}\right| .
$$

Let $B^{* \text { rot }}$ be a rectangle with slope $\theta_{k}$ such that

$$
\begin{gathered}
P_{1}\left(B^{* r o t}\right)=P_{1}(\widehat{R})=P_{1}\left(B^{*}\right), \\
B^{* r o t} \subset \widehat{R}_{\text {mid }}, \\
\left|P_{2,1}\left(B^{* r o t}\right)\right|=\left|P_{2,1}\left(B^{*}\right)\right| .
\end{gathered}
$$

Note that $\left|B^{*}\right|=\left|B^{* \text { rot }}\right|$. By Lemma 2, we have

$$
\left|B^{*} \cap R\right| \leq\left|B^{*} \cap R^{\infty}\right| \leq\left|B^{* r o t} \cap R^{\infty}\right|,
$$

and so we get

$$
\frac{\left|B^{*} \cap R\right|}{\left|B^{*}\right|} \leq \frac{\left|B^{* r o t} \cap R^{\infty}\right|}{\left|B^{* r o t}\right|} .
$$

By Lemma 3, this is equal to

$$
\frac{\left|\widehat{R}_{m i d} \cap R^{\infty}\right|}{\left|\widehat{R}_{m i d}\right|} \leq \frac{3|R|}{\left|\widehat{R}_{m i d}\right|}=\frac{9|R|}{|\widehat{R}|} .
$$

This implies

$$
\frac{\left|B^{*} \cap R\right|}{|R|} \leq \frac{9\left|B^{*}\right|}{|\widehat{R}|} \leq 27 \frac{\left|B^{*} \cap \widehat{R}\right|}{|\widehat{R}|}
$$


Hence

$$
\frac{|B \cap R|}{|R|} \leq 81 \frac{|B \cap \widehat{R}|}{|\widehat{R}|}
$$

and (12) is proved.

\section{Some Applications}

We can use Theorem 1 to give a simple proof of the following

Theorem 4. There exist positive constants $C$ and $\alpha$ such that for any set $\Omega \subset$ $\left[0, \frac{\pi}{4}\right)$ of cardinality $N>1$ one has

$$
\left\|M_{\Omega}\right\|_{L^{2} \rightarrow L^{2, \infty}} \leq C(\log N)^{\alpha},
$$

Proof. The proof is by induction on the number $\mathrm{N}$ of directions. It is clear that (14) holds for small N. Let us assume now that it is true for all $k<N$. We choose a subset $\Omega_{0}$ of cardinality $N^{1 / 2}$ so that the corresponding subsets $\Omega_{l}, l=1,2, \ldots, N^{1 / 2}$ have all cardinality $N^{1 / 2}$. Note that $N^{1 / 2}$ may not be an integer, but we may assume this with appropriate initial assumptions on $N$.

Then, by Theorem 1 ,

$$
\left\|M_{\Omega}\right\|_{L^{2} \rightarrow L^{2, \infty}}^{2} \leq C_{1} \sup _{1 \leq l \leq N^{1 / 2}}\left\|M_{\Omega_{l}}\right\|_{L^{2} \rightarrow L^{2, \infty}}^{2}+C_{2}\left\|M_{\Omega_{0}}\right\|_{L^{2} \rightarrow L^{2, \infty}}^{2} .
$$

By hypothesis, (14) holds for $M_{\Omega_{0}}$ and $M_{\Omega_{l}}, l=1, \ldots, N^{1 / 2}$. So we get

$$
\begin{gathered}
\left\|M_{\Omega}\right\|_{L^{2} \rightarrow L^{2, \infty}}^{2} \leq C_{1} C^{2}\left(\log \left(N^{1 / 2}\right)\right)^{2 \alpha}+C_{2} C^{2}\left(\log N^{1 / 2}\right)^{2 \alpha} \leq \\
\leq C^{2}\left(C_{1}\left(\frac{1}{2}\right)^{2 \alpha}+C_{2}\left(\frac{1}{2}\right)^{2 \alpha}\right)(\log N)^{2 \alpha} .
\end{gathered}
$$

Now we choose $\alpha$ appropriately so that $\left(C_{1}\left(\frac{1}{2}\right)^{2 \alpha}+C_{2}\left(\frac{1}{2}\right)^{2 \alpha}\right)$ is less than or equal to 1 and the theorem is proved.

Well known interpolation arguments show that from (14) one has the strong type estimate

$$
\left\|M_{\Omega}\right\|_{L^{2} \rightarrow L^{2}} \leq C(\log N)^{\alpha+\frac{1}{2}} .
$$

It is interesting to observe that the exponent $\alpha$ that we obtain in (14) depends only on the constants $C_{1}$ and $C_{2}$ in Theorem 1 . In particular, inequality (2) with $C_{1}=1$ would give the sharp exponent $\alpha=1 / 2$.

Our second application is an extension of a result by Sjögren and Sjölin [8]. We follow the notation introduced in Section 2.

Theorem 5. Let $\Omega_{0} \subset\left[0, \frac{\pi}{4}\right)$ denote the elements of a lacunary sequence $\left\{\theta_{l}\right\}_{l}$, say $\theta_{l} \leq \frac{1}{2} \theta_{l-1}$ and consider $\Omega_{l}, l=1,2, \ldots$ arbitrary sets with $\Omega_{l} \subset\left[\theta_{l}, \theta_{l-1}\right)$. Set $\Omega=\cup_{l \geq 0} \Omega_{l}$. Then the maximal function $M_{\Omega}$ has the property

$$
\left\|M_{\Omega}\right\|_{L^{2} \rightarrow L^{2, \infty}} \leq C \sup _{l}\left\|M_{\Omega_{l}}\right\|_{L^{2} \rightarrow L^{2, \infty}} .
$$


In particular, if each set $\Omega_{l}$ is given by the elements of a lacunary set as above (i.e., $\Omega$ is a double lacunary set), then $M_{\Omega}$ is of weak type (2,2).

The proof follows easily from Theorem 1.

Remark. At the time of submission of this paper for publication, the authors obtained a new result, similar to Theorem 1, but concerning now the strong type 2. Namely, under the hypothesis of Theorem 1 and with the notation given there, they showed that

$$
\left\|M_{\Omega}\right\|_{L^{2} \rightarrow L^{2}} \leq \sup _{1 \leq l}\left\|M_{\Omega_{l}}\right\|_{L^{2} \rightarrow L^{2}}+C_{2}\left\|M_{\Omega_{0}}\right\|_{L^{2} \rightarrow L^{2}} .
$$

This estimate yields a very simple proof of Katz's result, (1), with the sharp exponent, $\beta=1$. The details will appear in [2].

Also, the first author has recently extended these results to the case $p \neq 2$ in [1].

\section{References}

[1] A. Alfonseca, Strong type inequalities and an almost-orthogonality principle for families of maximal operators along directions in $\mathbb{R}^{2}$, to appear in J. London Math. Soc.

[2] A. Alfonseca, F. Soria, A. Vargas, An almost-orthogonality principle in $L^{2}$ for directional maximal functions, to appear in Proceedings of the Mount Holyoke Conference, Contemporary Mathematics, Amer. Math. Soc.

[3] J. A. Barrionuevo, A note on the Kakeya maximal operator, Math. Res. Lett. 3 (1996), $61-65$.

[4] A. Córdoba, R. Fefferman, On differentiation of integrals, Proc. Nat. Acad. Sci. U.S.A. 74 (1977), 2211-2213.

[5] N. H. Katz, Remarks on maximal operators over arbitrary sets of directions, Bull. London Math. Soc. 31 (1999), 700-710.

[6] Maximal operators over arbitrary sets of directions, Duke Math. J. 97 (1999), $67-79$.

[7] A. Nagel, E. M. Stein, S. Wainger, Differentiation in lacunary directions, Proc. Nat. Acad. Sci. U.S.A. 75 (1978), 1060-1062.

[8] P. Sjögren, P. Sjölin, Littlewood-Paley decompositions and Fourier multipliers with singularities on certain sets, Ann. Inst. Fourier (Grenoble) 31 (1981), 157-175.

[9] J.-O. Strömberg, Maximal functions associated to rectangles with uniformly distributed directions, Ann. of Math. (2) 107 (1978), 399-402.

Departamento de Matemáticas, Universidad Autónoma de Madrid, España.

E-mail address: angeles.alfonseca@uam.es

E-mail address: fernando.soria@uam.es

E-mail address: ana.vargas@uam.es 